\title{
La construcción de la ética de la empresa o ética de los negocios Segunda parte. Las formas a partir de la adopción de la economía*
}

\author{
Ricardo Cuevas Moreno**
}

\section{Resumen}

En este artículo explicaremos cómo la ética de la empresa o ética de los negocios se construye a partir de la contribución de la ciencia de la economía y de la administración. Son dos las formas que dicha articulación origina: "el empresario indispensable" $y$ "los valores morales son la clave del éxito". Entonces, en este trabajo se abordarán las formas propiamente dichas. Recordemos que el análisis de las formas descubre el contenido ideológico de la ética de la empresa; además, consideremos que el método de la economía política de Marx es la guía de nuestra investigación y de su exposición.

Palabras clave: dimensión ética, teorías de las organizaciones, teoría neoclásica, costos de transacción, racionalidad limitada, ética de los negocios, códigos éticos, decisiones estratégicas, ideología, historia del capitalismo.

\section{Introducción}

耳 $\mathrm{n}$ este artículo veremos cómo la ética de la empresa retoma los conocimientos de la economía y de la administración en su construcción, su discurso y sus recomendaciones. Esto desemboca en el dogma que sostiene toda la ética de los

\footnotetext{
* Este trabajo se conforma de dos partes. La primera se publicó en el número 216 de Contaduría y Administración.

** Investigador de la Facultad de Comercio y Administración de Tampico de la Universidad Autónoma de Tamaulipas. Correo electrónico: rcuevasfr@yahoo.fr
} 
negocios, el cual postula que los valores morales son la clave del éxito; este dogma cristaliza la articulación entre la ética, la economía y la administración de manera magistral del puño de los escritores de la ética de la empresa.

Además, este artículo explica, junto con el anterior, ${ }^{1}$ la fundación de la problemática propia de la ética de la empresa o ética de los negocios a la francesa. Con ello nosotros continuamos la explicación de cómo la ética de los negocios se construye, es decir, cómo ella es posible.

Recordemos que en el texto anterior afirmamos que el análisis de las formas de la ética de la empresa proporciona la prueba de que la ética de la empresa o ética de los negocios es una ideología del capital en el sentido de Marx y Engels (1982). Por forma entendemos la manera cómo se estructura el contenido de un proceso o fenómeno. En ese sentido, la ideología es la forma (representación) que adquieren las relaciones sociales de propiedad, de producción, de poder y de legitimación en la sociedad dividida en clases; asimismo, esta ideología toma la apariencia de conocimiento científico y se presenta como común a la sociedad, aunque en realidad ella postule los valores de una sola clase; de igual forma es una falsa conciencia con apariencia de verdad, puesto que perpetúa las relaciones económicas y sociales de explotación y dominio de una clase sobre otra ocultando su origen. En la práctica, la ideología impone las ideas y los valores de la clase dominante económicamente e intelectualmente, movilizando todos los recursos materiales y espirituales que dicha clase tiene a su disposición.

\section{La adopción de la economía por la ética de la empresa}

\section{a. El legado de Smith}

La ética de la empresa reposa sobre un conjunto de valores morales provistos por la sociedad capitalista. Nosotros sabemos que la integración de esos valores morales a la economía política remonta a la fundación misma de esta ciencia. Lo que nosotros llamamos la dimensión ética o la visión moral se encuentra en la base

\footnotetext{
1 "La construcción de la ética de la empresa o ética de los negocios. Primera parte. Las formas a partir de la aportación de la ética", Contaduría y Administración, FCA, UNAM, No. 216, mayo-agosto, 2005, pp. 119-141.
} 
de esta forma de ideología que es la ética de la empresa. La historia de la ética y las obras de Smith (1999 y 1991) Marx (1965a, 1982, 1965c, 1968b), Pribram (1986) y Hardt y Negri (2000), nos sirvieron para elaborar un esbozo de la genealogía de los valores morales del modo de vida capitalista, el cual nos ha servido de guía para nuestras investigaciones.

La aportación histórica y teórica del padre de la economía, en la explicación del orden económico y social, es una referencia obligada para la ideología del capital. Ésta olvida la crítica de esos valores hecha por Marx y Engels (1982) y Marx (1965c y 1968b) y que descubre, al mismo tiempo, el carácter científico e ideológico en la explicación de lo económico de Smith (Marx, 1995: 18-296). Al contrario, la ética de la empresa toma la dimensión ética del pensamiento de Smith como telón de fondo de todo su discurso: nos referimos a la idea de un sistema económico exento de contradicciones, en armonía (Smith, 1991: 81, 142, 422, 256), reino de la libertad, de la autonomía y de la independencia, naturales (Smith, 1999: $23,164,179,234,238,295-296)$.

Es esta dimensión ética provista por Smith y desarrollada por sus sucesores la primera aportación de la economía a la construcción de la ética de los negocios. Ahí, el mercado capitalista sólo puede establecer la armonía en despido de su historia real, donde una tal armonía no existe jamás sin la violencia organizada del Estado.

Esta articulación entre la ética y la economía política en la obra de Smith (Mathiot, 1990; Petkantchin, 1996; Sen, 1986; Novak, 1987; Marx, 1995) nos ha conducido ha establecer que en la base de su explicación del orden económico de Smith se encuentra su concepción del hombre y de la sociedad; además, uno de los legados más importantes del padre de la economía es esa dimensión ética o moral (Cuevas, 2003: 99-138).

\section{b. La empresa y la teoría neoclásica}

La ética de la empresa está construida a partir de los postulados del paradigma neoclásico; esto contiene una contradicción insuperable: la ética de la empresa preconiza los valores morales cuestionando la categoría de homo economicus, pero al mismo tiempo ella acepta el sistema de precios del marginalismo. Los conceptos y los principios de la teoría neoclásica son la segunda aportación de la economía para la ética de la empresa. 
Además, la ética de la empresa retoma algunos aspectos de la teoría económica sobre su teoría de la empresa o la firma. Uno puede hablar de una teoría económica de la empresa, utilizando el título del artículo de M. Koenig (Simon y Joffre, 1997); esta teoría razona a partir de los conceptos y los principios del paradigma neoclásico. A partir de una breve, pero compendiosa recapitulación de las teorías de la firma (Angelier, 1991) nosotros pensamos que existen dos factores importantes que impulsaron la evolución de la teoría de la firma: primero, el desarrollo histórico de la empresa capitalista; segundo, el carácter abstracto de la teoría de la empresa estándar para la explicación de los fenómenos. Ésta se funda sobre el jefe de empresa, la función de producción y la maximización de la ganancia para la asignación óptima de recursos en una situación de competencia perfecta. En efecto, según Koenig (Simon y Joffre, 1997: 938), el aumento de la extensión en el campo de investigación de la concurrencia imperfecta y las relaciones interfirmas conducen a profundizar la concepción de la empresa, pero conservando lo esencial del análisis neoclásico. La finalidad es construir una teoría de la empresa más cercana de los fenómenos de la realidad.

Las diferentes críticas sobre la concepción neoclásica de la empresa dan lugar a varias concepciones de la empresa. Así, considerar al empresario como un agente provisto de estrategia desemboca en la teoría béhaviorista y la teoría dictatorial de la firma; otra crítica sobre las limitaciones de la función de producción conduce a la teoría de los costos de transacción. De esta forma, R. H. Coase (1937) — según Koenig (Simon y Joffre, 1997) — responde a la cuestión de la supervivencia de las empresas en una sociedad donde el mercado prima y donde la racionalidad y la información de los agentes son limitadas.

Las teorias organizacionales de las firmas tratan de responder a los nuevos fenómenos de las grandes empresas capitalistas. El divorcio entre el capital propiedad y el poder, los conflictos entre los participantes de la empresa y los administradores, el consejo de administración y los accionarios son los temas de las teorías del control de la firma. En fin, las teorías sistemáticas de la empresa conciben la empresa como una organización que reagrupa los factores de la producción; además, esta organización es una totalidad de relaciones y de subsistemas que reúnen las relaciones internas y externas de la firma. Esas teorías sintetizan las contribuciones de los economistas y los administradores dando una visión de la empresa que pretende corresponder más a la realidad actual (Angelier, 1991). 
Sin embargo, nosotros pensamos que seguir la pendiente del análisis de los conflictos entre todos los participantes de la empresa conduciría indefectiblemente al origen de las relaciones de explotación y de poder en la empresa. Al contrario, la ética de la empresa prefiere definir a ésta con base en la teoría neoinstitucional de costos de transacción que le permita evitar profundizar en esos conflictos.

Según Koenig (Simon y Joffre, 1997), el punto de vista de la teoría neoinstitucional reúne los elementos de las teorías neoinstitucionales de T. Veblen, J.R. Commons y W.C. Mitchell de los años 1920 y 1930, así como ciertos conceptos y mecanismos de la teoría del comportamiento de H. Simon y J.G. March (1979), R.M. Cyert (1963) y O.E. Williamson $(1979,1994)$. Este último autor, principal representante de este acercamiento, concibe el espacio económico como una red de contratos bilaterales; es decir la empresa es una red centralizada y regulada de contratos específicos cuyo objetivo fundamental es la ganancia. Asimismo, Williamson establece que los contratos son específicos e incompletos en razón de la racionalidad y de la información limitada de los agentes; la función primordial de estos contratos es asegurar la coordinación de la producción de la empresa; su forma general y su práctica continua reduce la incertidumbre en un universo económico complejo e incierto. Eso se traduce en una reducción de los costos de previsión por la adquisición de reglas de decisión, de formas de funcionamiento y de códigos que hacen más fácil la adquisición de información y la comunicación. En fin, la adopción y la adhesión a esas reglas y a esos códigos aseguran el establecimiento de un lenguaje común y una cada vez mayor extensión de los límites de la racionalidad en la toma de decisiones de los agentes (Simon y Joffre, 1997: 3310, 3318-3319).

En suma, la tercera aportación de la economía a la ética de la empresa es la definición de empresa como una red centralizada de contratos específicos. Esta definición merece por nuestra parte una observación con la finalidad de subrayar la función que ésta desempeña en la ética de la empresa como forma de ideología.

En primer lugar, el contrato se establece entre hombres libres, autónomos; ellos empeñan sus voluntades en términos de igualdad. Ese compromiso implica responsabilidades y el cumplimiento de ciertas obligaciones. Según los escritores de la ética de la empresa, eso implica la necesidad de las cuestiones morales para los participantes en la empresa. 
Pero la igualdad de los contratantes es aparente. Marx (1965c) muestra cómo, a partir del intercambio más simple de mercancías contra el dinero, el contrato es la expresión de voluntades de los contratantes y la de su propiedad privada sobre sus mercancías; pero cuando el capital prima sobre la sociedad, las leyes de apropiación simple de mercancías se transforman en leyes de apropiación del capital (Marx, 1965c: 619-620, 1088-1090). La aparente equidad de los intercambios entre las clases sociales o capas de clase (en el sentido moderno del término) y la clase propietaria del capital (en cualquiera de sus formas) esconde la extracción de la ganancia de una clase sobre otra. La empresa como simple nudo de contratos enmascara las relaciones de autoridad y de dominación en los diferentes procesos de la producción y de la distribución social. He aquí el servicio que una definición de la empresa como nudo de contratos provee a la ética de la empresa y a toda ideología del capital.

Así, el Centro de Jóvenes Dirigentes (CJD) que forma parte de la estructura de la reflexión ética de la empresa en Francia establece una definición de la empresa que parece más adecuada para la época actual:

La empresa es un grupo de hombres y de mujeres, reunidos en el marco de un proyecto común, para crear el nexo social, por intermedio de una producción de bienes y de servicios materiales o inmateriales, bajo presiones económicas y sociales, en interacción con el medio ambiente. (Claude, 1998: 124)

En ese caso, la posibilidad de considerar las posiciones de los participantes en la empresa a partir del origen de los contratos es borrada por el proyecto compartido entre hombres y mujeres frente a las presiones económicas y sociales.

Pero a nuestra crítica se opone la objeción inmediata de la veracidad de las definiciones de empresa retomadas por la ética de la empresa; sin embargo, se trata de una verdad que deja de lado los elementos sobre los cuales ellas mismas se fundan. Es por esa razón que la ética de la empresa deviene en ideología del capital. Así, la empresa es una unidad de producción autónoma destinada a la producción de bienes y servicios, a la combinación de factores de producción y a la obtención de ingresos para sus participantes. También es un nudo de contratos entre los propietarios del capital y los desposeídos de éste; la existencia de esos dos contratantes y las relaciones de propiedad son las condiciones sine qua non para el establecimiento de los contratos y su reiteración. Uno constata entonces que las condiciones sobre las cuales los contratos reposan van más allá de la libertad, de la autonomía, o de la responsabilidad. Al momento de conside- 
rar las cosas desde ese punto de vista, los valores morales como la libertad, la igualdad o la justicia acusan su carácter histórico para los contratantes y, por tanto, transitorio y limitado en el tiempo.

\section{c. El empresario indispensable}

La historia del surgimiento del capitalismo en la interpretación de Weber (1967) es la cuarta aportación del conocimiento a la ética de la empresa. Esta aportación se encuentra entre las fronteras de la historia del capitalismo, la historia de las religiones y la economía. Según Weber (1967), el capitalismo tiene un origen ascético; el nuevo éthos del capitalismo está encarnado en el empresario racional, metódico, portador de las cualidades de la religión ascética. Estas afirmaciones han devenido en un filón inagotable para la ética económica en general y para la ética de la empresa en particular. Se trata de la explicación del origen moral del capitalismo y de la existencia indispensable del empresario capitalista. Ahora veamos la forma que toma ese filón inagotable en la ética de la empresa.

Hacia 1997 en el Coloquio ética de los negocios: de la ética del empresario al derecho de los negocios se puso en relieve la siguiente pregunta: ¿Es posible y válido usar la noción ética del empresario? (Naudet, 1997). Para responder a esa cuestión, los autores de esa obra colectiva parten de la serie de valores morales que son el marco de la sociedad actual. Ellos afirman que esos valores se cristalizan en la política y el derecho; los valores afectan en general la vida de los individuos y en particular la vida de la empresa. En ésta, los valores morales ocupan un lugar tan importante como lo económico. En dicho coloquio también se señaló que la reflexión sobre la ética de la empresa y la historia del pensamiento económico han olvidado la importancia del empresario, su responsabilidad y la serie de cualidades morales que éste debe tener.

Uno de los participantes de este Coloquio, Salin, en su conferencia l'Entrepreneur et l'Ethique (Naudet, 1997: 117) trató de fundar la ética del empresario a partir de las tres ideas siguientes:

Primera, la empresa es ante todo un nudo de contratos; un conjunto de relaciones personales, un conjunto de relaciones sociales cuyo objetivo es la ganancia, la que a su vez es uno de los objetivos de la empresa, pero no el único. Los diferentes individuos que intervienen comprometen su responsabilidad usando 
su libertad individual; de ahí el fundamento de la ética en la empresa y, en particular, de la ética del empresario.

Segunda, el empresario es quien toma las decisiones en la gestión de la empresa; sobre él pesa la carga de la responsabilidad adquirida gracias a la libertad para que la empresa alcance sus objetivos. Esta libertad no puede existir sin los fundamentos primeros de la sociedad que son los derechos naturales del individuo; el otro fundamento de la ética del empresario es el sistema de normas universales: normas de libertad y de propiedad en un universo incierto.

Por consiguiente, la responsabilidad del empresario es la clave que legitima la obtención de la ganancia. En efecto, el empresario asume la responsabilidad, los riesgos de los negocios de la empresa; la ganancia es la recompensa de ese esfuerzo. Por su parte, Salin piensa que, frente a una visión "instrumentalista" y utilitarista del empresario, la ganancia es la recompensa por tomar buenas decisiones, por proveer un servicio a los consumidores. Además, el empresario efectúa una función social mayor que consiste en tomar a cargo el riesgo porque él es un creador. Con esto, pensamos nosotros, la ética de la empresa renueva las teorías de la abstinencia y los servicios en origen del capital y de la ganancia criticadas por Marx. (1965, 1995: 1095-1104).

Por otro lado, la ética del empresario no estaría completa sin las cualidades morales e intelectuales necesarias para el empresario. Por ejemplo, Ortiz-Ibarz, en su conferencia La prudence de l'Entrepreneur (del mismo coloquio), piensa que la administración, la toma de decisiones y el control de la empresa hacen necesario poseer una cultura general; además, tener una buena memoria, aprender a escuchar, tener el sentido del humor, y una buena educación son otras cualidades que se unen a la cultura general. (Naudet, 1997: 123-124,126-128 y 132-136).

Una visión más extrema de la indispensabilidad del empresario es la de Etchegoyen (1990) quien lo considera como la encarnación misma del alma de la empresa. En este sentido, el empresario sería la encarnación del principio, de esta cosa que no se pude definir ni conocer y que anima la empresa. El empresario junto con el management, la estrategia, la cultura y la comunicación constituyen el alma de la empresa (Etchegoyen, 1990: 28, 33-37, 79, 94, 121, 133, 140-150).

De este modo, pensamos, la ética de la empresa provee una versión idealizada y falsa del empresario. En esa tarea, ella debe olvidar la historia del capitalismo y la 
historia reciente de las empresas, donde la autogestión de los grupos de trabajo hace inútil la existencia del capitalista en el proceso directo de la producción y la administración. Es la tendencia histórica del capital, que Marx (1965c: 1237-1240) observaba, la que se cumple en la actualidad: una creciente socialización de la producción frente a la apropiación privada de los frutos del trabajo. Aunque el resultado de ese trabajo puede ser inmaterial; éste pertenece al capitalista, pues él obra de acuerdo con las leyes del mercado y con los contratos legales, expresión de esas leyes. En tanto que propietario, el capitalista puede utilizar ese conocimiento en provecho de su empresa o venderlo a cualquiera, pues al igual que la mercancía le pertenece por derecho, aunque él no lo haya creado.

Por tanto, la ética del empresario olvida una característica primordial del capitalismo formulada por Marx (1965a: 164), (1965c: 859-999, 948): el capital pone a su servicio todos los conocimientos, todas las fuerzas naturales, físicas y mentales de la sociedad del presente y del pasado para la producción de ganancia. Es el saber humano acumulado que el capital toma a su servicio. Por consecuencia, pensamos, la idea que el empresario es un creador indispensable por el mundo capitalista funciona como ideología dando una visión falsa del rol del empresario para la vida económica y social. El empresario indispensable es una manera de legitimar el primado de la burguesía sobre el resto del organismo social; es un conocimiento, un servicio gratuito que el economista, el administrador, el sociólogo, el abogado y el filósofo proveen al capital.

\section{d. El dogma fundamental de la ética de la empresa}

Llegamos al dogma que da validez a la ética de la empresa. Este dogma es su carta de naturalización, su razón de ser, su resplandor, su atractivo. La razón por la cual la ética de la empresa es digna de atención para el capital y la opinión pública es que los valores morales son la clave del éxito de la empresa. Al igual que un dogma religioso nadie duda de la validez de esa afirmación; pero al momento que uno descubre la base real sobre la cual ese dogma se levanta uno comprende el carácter mistificador que ese producto del hombre contiene. Por consiguiente, para nosotros, ese dogma resume y cristaliza esa forma de ideología del capital. Al momento en que descubrimos su contenido y la forma que éste toma, su carácter mistificador desaparece, pero eso no implica que los creyentes lo abandonen en tanto que dogma de fe; al igual que el creyente, la ética de la empresa tiene poderosas razones de no abandonar el dogma, el cual le provee de su razón de ser. 
La ética de la empresa ha sido un tema importado por los patrones franceses, y exportado por las empresas estadounidenses. Aunque existe en la actualidad una ética de la empresa a la francesa, los empresarios franceses también han importado el dogma referido, carta mayor de esa ideología; a pesar que antaño Fayol (1981: 190,193) establecía la importancia de los valores morales para la administración de la empresa y su éxito.

La afirmación: los valores morales son la clave del éxito de la empresa toma algunas variantes y formulaciones, que pueden clasificarse en:

1. Las que consideran que los valores morales son la clave del éxito en la toma de decisiones, dígase moralmente válidas y eficaces; en ese caso la adopción de los valores morales de la empresa es hecha por los que toman decisiones importantes: los administradores de los cuadros superiores.

2. Las que consideran que los valores morales deben ser adoptados por todos los participantes de la empresa; en ese caso todos los hombres que trabajan deben identificar su proyecto personal con el de la empresa.

3. Una combinación de ambas formulaciones. Los valores morales son necesarios para los dirigentes en la toma de decisiones y para que todos los individuos identifiquen su proyecto individual al proyecto compartido de la empresa.

Es cierto que en esos casos lo que permanece es la necesidad de la adopción de "los valores morales de la empresa" de parte de los hombres que trabajan para ella. De ahí proviene la necesidad de hacer operatoria, de aplicar, de traducir esos valores morales de la empresa en la administración. La puesta en práctica es el objetivo fundamental y la consecuencia necesaria de la promesa del éxito; es decir, convertir a la ética en un útil de gestión a pesar de que ciertos autores como Even-Granboulan (1988), Etchegoyen (1990) u Orsoni (1999) consideran que un uso tan pragmático abre la puerta a la manipulación moral sobre los hombres que trabajan. Ciertamente, nosotros pensamos que la ética de la empresa transforma la ética en un útil para la acumulación de las ganancias y el aumento de la eficiencia mediante la movilización de los hombres. Esto es un hecho de los más naturales dentro del marco de la sociedad capitalista.

La adopción de los valores morales de la empresa de parte de los hombres que trabajan para ella es el punto crítico; ésta debe hacer posible el éxito común de la empresa y de los hombres. El otro resultado prometido es el florecimiento de las 
potencialidades de los participantes; sin embargo, ésa es la forma exterior que olvida ciertos aspectos y procesos reales en la transformación de una economía industrial hacia una economía de la informatización y de la comunicación; por ejemplo, según l'Empire (Hardt y Negri, 2000), ese fenómeno se expresa en cambios profundos en la estructuración de las relaciones entre el proletariado moderno, el capital y las empresas en el nivel mundial. Un hecho de los más importantes de ese cambio en el paradigma productivo es la modificación en la forma de vida; nos referimos al primado de lo que esos autores llaman el trabajo inmaterial, nuevo avatar de la producción de plusvalía sobre el trabajo de la agricultura y la industria (Hardt y Negri, 2000: 303-307, 319-333, 359363). Por consiguiente, pensamos nosotros, que el dominio de esa nueva forma de economía capitalista requiere de trabajadores adecuados para su realización; se trata no solamente del primado de la producción inmaterial, sino de la adecuación de la subjetividad de los hombres comprometidos en esta producción. Es así que la comunicación/informatización y la ideología del capital (de la cual los valores morales para la empresa son una parte) son un universo por descubrir; en cambio para los escritores de la ética de la empresa, ese universo es simplemente un supuesto de investigación. En breve, el compromiso que la empresa solicita a los hombres que para ella trabajan está lejos de limitarse a las exigencias de un trabajo más autónomo, al comportamiento moral y a la promesa del florecimiento personal.

En lugar del análisis de los fenómenos, la ética de la empresa toma su forma exterior describiendo su existencia. Dicho análisis es sustituido por otra problemática; por ejemplo, la idea central del libro Le prix de l'excellence de Peters y Waterman (1983) es que los salariados son más productivos si se identifican con los valores de la empresa. Una formulación más fina de esta problemática es manifestada algunos años después en L'éthique au service du management (Claude, 1998): partiendo del supuesto de la sociedad democrática y liberal en la cual existe una institución llamada empresa ¿cómo la ética puede servir al management de la empresa performante? Para dar respuesta a esta pregunta es necesario responder antes a la siguiente: ¿Cómo el individuo puede llegar a identificar su proyecto personal de vida con el éxito de la empresa? De esta manera el problema es explicar, mediante la ética, la economía y la administración, la adopción de los valores morales por parte del individuo. Al contrario, nosotros establecemos la pregunta: ¿por qué a partir de los años 1980 la necesidad de gestionar la subjetividad del proletariado moderno se convierte en un asunto central para la producción y la legitimación de la empresa? 
Aún más. Uno puede ubicar la aparición de lo que nosotros llamamos el dogma fundamental de la ética de la empresa a partir de la aparición de los libros de Blanchard y Peale (1988), Solomon y Hanson (1989) y en Francia Gélinier (1991). Así, la idea fundamental del libro Ética y management es que un sólido código moral es la primera condición del éxito de la empresa. Para hacer operatoria la ética en la empresa, Blanchard y Peale (1988: 24, 26, 28, 31) proponen un test ético y la fuerza de la costumbre para establecer el comportamiento moral en la toma de decisiones:

Nosotros estimamos que un sólido código moral es el primer elemento del éxito de toda empresa. Nosotros pensamos que los managers que obran según los principios morales son los managers que ganan. (Blanchard y Peale, 1988: 12)

Según Solomon y Hanson, los negocios son más que el crecimiento y la búsqueda de beneficio. Ellos son una forma de vivir, en la cual la sobrevivencia y la prosperidad dependen de la participación de los practicantes. De esta forma, para esos autores, la ética es el fundamento mismo de los negocios (Daigne, 1991: 97).

Según Claude (1998: 129-130), los valores aparecen como el trazo de unión entre el individuo y la empresa. Existe un acuerdo preestablecido de valores morales, los cuales permiten la armonía entre el proyecto personal y la performance de esta institución económica y social que es la empresa. Éstos explican, según Claude, la trama de relaciones que constituyen la empresa y la sociedad capitalistas; entonces, pensamos nosotros, por encanto el origen de las relaciones de producción es substituido por la existencia de los valores morales.

Una forma muy sutil de esa sustitución es la de Archier y Serieyx (1984); para ellos, la empresa del primer tipo es la empresa inspirada en la doctrina de Taylor (1986, 1911), en la cual la organización es tiránica y piramidal; ella está animada por los principios de jerarquía, de disciplina y de rigidez. La empresa del segundo tipo es la del aumento de la productividad gracias a la automatización. La empresa del tercer tipo se caracteriza por su estructura inversa y ella está animada por los principios de confianza y de flexibilidad (Le Tourneau, 2000: 154).

Establezcamos que la clasificación de Archier y Serieyx establece dos criterios diferentes: primero, el crecimiento de la capacidad productiva del trabajo para lo que ellos llaman la empresa del primer tipo y la empresa del segundo tipo; en seguida, el criterio de clasificación cambia y es sustituido por otro de carácter moral (subjetivo) unido a otro (objetivo): la confianza y la flexibilidad para la 
empresa del tercer tipo. Entonces, la clasificación contiene una falta de lógica y de objetividad porque anula las transformaciones reales de la emergencia de la empresa del tercer tipo como lo es la revolución en la informática y las comunicaciones y las sustituye por las relaciones morales que pretenden cambiar las relaciones de producción. De esta forma la ética de la empresa toma forma.

Por otro lado, según Gélinier (1991) frente a la corrupción mundial es necesario recordar las raíces morales y éticas de las actividades humanas, especialmente de los negocios. Para ese propósito no es solamente necesario justificar la necesidad de la ética, sino de proponer los principios y la estructura institucional que hagan posible la práctica de esta ética particular. Gélinier piensa que eso acrecentará la confianza, los valores y la lealtad en el mundo de los negocios; con su libro pionero los valores de la empresa como clave del éxito toman forma de aplicación y sistemática para las decisiones estratégicas (Gélinier, 1991: 19-47, 85-86, 90-92, 166-169).

De esta manera Gélinier traza el camino seguido por los escritores de la ética de la empresa en Francia. Desde entonces la aplicación de la ética o de la moral de los negocios debe tomar la forma de una carta o de un código ético que oriente los deberes, las responsabilidades y en su caso castigue a los infractores; esto permitirá a la empresa ser más competitiva y conseguir el éxito. En un sentido más amplio, se trata de la organización de la ética de los negocios en Francia y la creación de la estructura jurídica para su ejercicio. Gélinier establece los principios y las recomendaciones para que los patrones puedan poner en obra la ética de los negocios (Gélinier, 1991: 155-156, 166-169, 175, 178-180).

Una aplicación a la esfera financiera retomando en lo fundamental las ideas de Gélinier se encuentra en Daigne (1991: 97-109, 112). Para él, la ética de los negocios debe convertirse en un motor del desarrollo y de la eficiencia. La adopción de los valores morales de la parte de los administradores es, entonces, la clave de esta ética particular (Daigne, 1991: 56, 97, 100, 102, 109, 256).

\section{e. El dogma de la ética de la empresa y la ley del valor}

Gélinier $(1991: 10,69)$ establece que la práctica de los valores morales como la confianza produce un valor agregado al valor del producto; desde entonces esta idea es retomada infinidad de veces para probar que los valores morales son fuente de valor. Ciertamente, la promesa del éxito gracias al ejercicio de los valores 
morales en la empresa debe mostrar sus ventajas haciendo crecer el capital; de otra forma, la clase capitalista no estaría atraída por la ética en los negocios.

Uno sabe que la ética de la empresa razona a partir del sistema de precios del paradigma dominante en economía; eso es un supuesto para la ética de la empresa. Para nosotros, esa manera de proceder, a partir de la teoría subjetiva del valor, lejos de ser algo simple, es un punto delicado y merece un análisis. Así, los valores morales tales como solidaridad, lealtad, libertad o cualquier otro valor, añaden valor al producto. Por ejemplo, Le Tourneau, en su conferencia L'éthique des contrats internationaux establece:

Por tanto, a largo plazo, la moral produce valor agregado: asegurando una ventaja competitiva, ella es un útil estratégico de primer orden, sobre todo en los negocios internacionales donde los participantes, menos numerosos, se conocen. (Naudet, 1997: 228).

En efecto, a partir de la teoría subjetiva del valor cualquier virtud puede contribuir a la formación del producto; sin embargo, la manera como eso sucede con los valores morales no es explicada; en ese caso, nosotros pensamos, la disciplina de la administración puede proveer una idea sobre cómo ese proceso de agregación de valor pasa.

La administración moderna de recursos humanos pone en relieve la importancia de la gestión de los hombres y de la comunicación. Durante el reclutamiento, los candidatos son evaluados a fin de escoger los que tengan mayores posibilidades para integrarse a los valores, al proyecto y a la cultura de la empresa; una vez contratados, los nuevos reclutas pueden ser formados en el aprendizaje del trabajo y de la ideología de la empresa; de esta manera, la empresa asume los gastos necesarios para la integración del personal a sus valores morales.

Supongamos que la gerencia solicita que el valor moral de la responsabilidad sea adoptado por los hombres que trabajan para la empresa. Desde ese supuesto, los empleados y los obreros asistirán puntualmente al trabajo y efectuarán sus actividades en los tiempos prescritos, inspirados por el valor moral de la responsabilidad. En la práctica, la asistencia continua al trabajo evitará el desperdicio del tiempo y de recursos posibles, que tendrían lugar en la ausencia del valor de la responsabilidad; pero ahorrar es diferente que añadir, o mejor aún, es diferente de multiplicar el valor económico; en ese caso, la responsabilidad no añade nunca un valor económico al producto. 
Otro ejemplo es el administrador que de manera responsable y honesta evita malversar los fondos de una compra fraudulenta con un proveedor; en ese caso, no es la responsabilidad o la honestidad quienes añadirían un valor económico al producto; entonces, la empresa obtendrá la ganancia normal suponiendo una marcha normal de los negocios.

Sin embargo, uno creerá que los valores morales añaden valor al producto porque la empresa gasta dinero para la administración de recursos humanos y para la comunicación; además, ciertos fenómenos pueden apoyar esa idea. Supongamos que la responsabilidad se traduce en empeño en el trabajo, no de manera particular sino para la empresa en su conjunto; en ese caso, la ganancia crecerá; pero nosotros pensamos que la intensidad del trabajo es el verdadero elemento que explica el aumento de valor y no algún valor moral imaginado por el autor de la ética de la empresa. Esta perspectiva nos conduce a considerar las cosas desde la teoría objetiva del valor; expliquemos, entonces, la idea según la cual los valores morales añaden valor económico al producto a partir de esa perspectiva.

La teoría del valor de Marx (1965c: 561-619, 897-899) establece que el valor de las mercancías es una relación social de producción entre el tiempo de trabajo de la sociedad, las necesidades sociales y la masa de productos. Esos elementos son comprendidos desde el marco de la producción mercantil simple y la producción capitalista de mercancías; es decir, en la producción de productores privados autónomos e independientes, la división social del trabajo y los intercambios mediante la moneda.

Recordemos, además, que la ley del valor establece cuanto del tiempo de trabajo de la sociedad debe dedicar para satisfacer cada una de sus necesidades de mercancías; es una ley ex post facto, que se expresa a través de las variaciones del precio de mercado. La sociedad no conoce de avance exactamente el quantum de las mercancías que ella produce, sino que después de realizados los cambios. Reducir esa separación es una preocupación y un quehacer fundamental; así, el marketing del gran consumo trata de reducir la brecha entre la necesidad social solvente y la cantidad de mercancías producidas; entonces, la ley del valor no es solamente el tiempo de trabajo social necesario para la producción de mercancias, como los marxistas lo creen; ella es también una relación de producción y de distribución de la riqueza social (Marx, 1965d: 1304, 1308, 1452, 1457).

Además, la ley del valor contiene infracciones enunciadas por Marx. Se trata de la formación de precios que evitan la determinación por la ley del valor; así, los 
precios de monopolio, como el precio de la renta de la tierra, es un precio que viola la ley del valor, pero al mismo tiempo supone la producción de mercancías como forma dominante de la producción social. El autor de El Capital, en el libro III, los llama los precios irracionales (Marx, 1965d: 1316, 1389).

Así, objetos que no son productos del trabajo adquieren la forma precio; por ejemplo, la confianza, el amor, la lealtad. En efecto, según Marx, para que un objeto tome la forma precio, es suficiente con que él sea enajenable y posible de monopolizar; por esa razón, objetos de la naturaleza como los ríos o el suelo adquieren la forma precio, aunque ellos no sean originariamente productos del trabajo social.

Para el escéptico recordemos que en la actualidad el agua se vende al igual que un poema de Jim Morrison. Al economista le sorprende que esos objetos tomen la forma precio, se diga que tiene un valor y que circulen como mercancías; mas para evitar las complicaciones, el economista recurre a la teoría subjetiva del valor, la cual le dice que no es necesario determinar los precios mediante el tiempo de trabajo socialmente necesario, ni a la magnitud del valor por la cantidad de ese trabajo, ni que esa cantidad toma la forma de precio, mucho menos que la forma precio contiene en germen la irracionalidad.

En la teoría económica de Marx, ni la confianza, ni la lealtad crean valor; ni stricto sensu ni lato sensu; ellas no pertenecen a la formación del valor o de la ganancia; ¿cómo entonces esos valores morales pueden afectar dicha formación de valor?

Para la teoría subjetiva del valor, el valor de la confianza u otro valor moral, puede determinarse por la voluntad pura y simple del individuo racional; en consecuencia, es posible hablar de valor moral y valor económico, de precio moral y precio económico, como si se trataran de precios de una misma naturaleza. Al contrario, la teoría del valor de Marx (1965c: 640-642) explica las razones por las cuales los valores morales pueden tomar la forma precio; se trata, lacónicamente, de la distinción entre la forma de precios irracionales y la forma de precios racionales.

En efecto, los valores morales y los valores económicos son dos cosas diferentes; las actividades prácticas y transformadoras que les dan vida pertenecen a dos mundos distintos. La ética de la empresa al igual que la teoría subjetiva del valor olvidan esa distinción, de tal suerte, uno pude identificar los valores morales y los valores económicos; para nosotros, eso es una operación artificiosa. Por consecuencia, el dogma que establece que los valores morales son éxito de la empresa 
se funda sobre la teoría subjetiva del valor porque ésta le permite comparar los valores morales y los valores económicos borrando su diferencia y su origen a través de la forma precio.

En suma, es claro que el dogma: los valores morales de la empresa son la clave del éxito implica serias objeciones que ningún autor ha notado antes que nosotros. Esas objeciones nos conducen a sostener que la ética de la empresa o la ética de los negocios no puede demostrar que los valores morales producen verdaderamente valor o asegurar un éxito a largo plazo.

Una versión extrema de ese dogma difundido por los escritores de la ética de la empresa y los medios de comunicación masiva (Leroy, 2002) conduce a creer que los valores morales explican la existencia de la empresa y la estructura económica de la sociedad. A este respecto podemos proponer la siguiente clasificación: (a) Una versión extrema moderada trata de establecer que los valores morales son tan importantes como la eficiencia en la administración o la existencia objetiva del sistema productivo; pero esta versión da un lugar importante, a largo plazo, a los valores morales para el éxito. (b) Otra versión, también moderada, establece que la ética de la empresa o ética de los negocios es un arma para mejor gestionar la empresa (Orsoni, 1999); en ese caso, a justo título, el comportamiento moral en la empresa puede contribuir a las economías en el empleo del capital circulante (los robos, los pasajeros clandestinos), y de esta manera conservar la ganancia, pero no acrecentarla. (c) Una visión crítica, como la que nosotros defendemos, da un lugar e importancia que los valores morales poseen verdaderamente para la empresa y el modo de vida capitalista.

Un análisis histórico hará evidente que los valores morales para la ética de la empresa tienen una función más importante, pero diferente a la aceptada por el dogma: los valores morales son la clave del éxito. Landier $(1991: 264,270)$ piensa que la pertinencia de la reflexión ética en el nuevo management proviene del hecho que la empresa solicita al individuo comprometerse en un proyecto común: el de la empresa. Esas afirmaciones conducen a razonar sobre dos relaciones: primera, la de la vida personal y la vida dentro del espacio público; segunda, la del tiempo de trabajo y el tiempo del ocio. El hecho que la empresa demande e incida de manera creciente en esas relaciones modificando su magnitud, conduce, sin duda, a la búsqueda del sentido y, por tanto, a la ética. Además, pensamos, eso conduce a una reflexión de carácter ideológico y político de la empresa cara a cara a la sociedad. Ahí, el rol del management participativo, de la información y de la 
comunicación en la administración de la empresa y de la sociedad nos ayudarán a encontrar el lugar que la ética de la empresa o ética de los negocios tienen verdaderamente en tanto que forma de ideología del capital.

\section{Resumen y conclusión}

1. En este artículo explicamos cómo la ética de la empresa o ética de los negocios retoma de la economía su conocimiento para fundar su discurso. La historia del pensamiento económico en la obra de Smith le provee la dimensión ética o la visión moral sobre la cual se basa esta forma de ideología.

2. La obra de Weber sobre el origen del capitalismo es utilizada para fundar, una mal llamada "ética del empresario", la cual deviene - según nosotros - en una apología de la necesidad indispensable de la clase capitalista para la producción y la vida social.

3. La otra fuente retomada por la ética de la empresa es la teoría neoclásica y sus principios. Nosotros hemos puesto en evidencia las contradicciones que eso entraña: (a) abogar por la integración de las cuestiones morales en la economía y en la empresa, permaneciendo encerrado dentro del sistema de precios neoclásico; (b) utilizar la teoría subjetiva del valor para comparar los valores morales y los valores económicos sin ninguna distinción, pensando que uno y el otro añaden valor. Más este quid pro quo proviene de la teoría subjetiva del valor, pero la ética de la empresa o ética de los negocios lo reproduce.

4. La concepción de la empresa como una red de contratos es otro préstamo usado por la ética de la empresa. La crítica fundamental de nuestra parte es que esa concepción oculta las relaciones de dominación, de poder y de legitimación en la empresa deviniendo en una base para fundar esa ideología del capital que es la ética de la empresa.

5. Los elementos señalados en los puntos precedentes permiten establecer una problemática: nos referimos a la identificación del proyecto y del éxito de la empresa con el proyecto personal de los hombres. En otras palabras, ¿cómo los hombres pueden aceptar y adoptar los valores morales propuestos por la empresa? En suma, ¿cómo la ética puede convertirse en un útil de administración? A esa problemática nosotros oponemos otra: ¿Por qué desde los 
años 1980 del siglo pasado la necesidad de gestionar, de administrar la subjetividad del proletariado moderno se convierte en un asunto crucial para la producción y la legitimación de la empresa?

6. Todos estos elementos se cristalizan en el dogma fundamental de la ética de la empresa: los valores morales son la clave del éxito; entonces, todas las objeciones que hemos investigado en las páginas precedentes invalidan ese dogma, descubriendo su carácter ideológico en el sentido de Marx y Engels (1982). Pero este descubrimiento no abolirá la existencia de dicho dogma ni de la ética de la empresa o ética de los negocios; ni tampoco abolirá el hecho que seguirá siendo repetido por los espíritus prisioneros del mundo de las apariencias o ligados a los intereses de la clase burguesa.

7. En fin, vemos que las contribuciones al conocimiento hechas por la ética de la empresa contienen contradicciones insuperables. Este ensamblado que es la ética de la empresa, para existir, debe hacer un arreglo en las interpretaciones de los filósofos, economistas y sociólogos; tomar a préstamo los conocimientos de diferentes corrientes de la economía y de la administración para tener una apariencia de coherencia interna y externa. Esto es indispensable, pues de otra manera la aplicación práctica de la ética de los negocios sería sumamente limitada o imposible. Al contrario, el cumplimiento de esa inversión del pensamiento provee a la ética de la empresa o ética de los negocios su validez, la coherencia indispensable para funcionar como ideología y contribuir a la legitimidad de la empresa misma y en el espacio público. De esta forma la ética de la empresa o ética de los negocios se presenta como ideología para todos y no de una clase o fracción de clase particular.

\section{Bibliografía}

Alix-Desfautaux, E. et Joffre, P. ; "Coût de transaction", en Simon, Y. et Joffre, P. (sous la direction); Encyclopédie de Gestion, Paris, Economique, $2^{\mathrm{e}}$ édition, T. I, p. 751

Angelier, J-P ; Economie Industrielle, Grenoble, Presse Universitaires de Grenoble, Pug, 1991 
Archier, G. et Sérieyx, H. ; L'entreprise du 3e type, Paris, Editions Seuil, 1984

Bergmann, A. ; "Ethique et gestion", en Simon, Y. et Joffre, P. (sous la direction) ; Encyclopédie de Gestion, Paris, Economique, $2^{\mathrm{e}}$ édition., T. II, p. 1239

Blanchard, K et Peale N.V ; Ethique et management. Réussir en restant intègre, Paris, Traduction par Marnat S ; Les Editions d'Organisation, 1988

Charreaux, G. ; "Gouvernement de l'entreprise", en Simon, Y. et Joffre, P. (sous la direction) ; Encyclopédie de Gestion, Paris, Economique, $2^{\mathrm{e}}$ édition, T. II, p. 1652

Claude, J-F ; L'éthique au service du management, Paris. Les Éditions LIAISONS, 1998

Cohen, E. ; "Epistémologie de la gestion", en Simon, Y. et Joffre, P. (sous la direction) ; Encyclopédie de Gestion, Paris, Economique, $2^{\mathrm{e}}$ édition, T. I, p. 1158

Cuevas Moreno, R. ; Éthique de l'entreprise capitaliste: Essai sur "L'éthique de l'entreprise " en France et l'idéologie, France, Thèse de Doctorat en Sciences de la Gestion, Université de Corse Pascal Paoli, Institut d'Administration des Entreprises, 2003

"La construction de l'éthique de l'entreprise. Première Partie. Les formes à partir de l'adoption de l'éthique", en Contaduría y Administración, FCA, UNAM, No. 216, México, mayo-agosto, 2005

Daigne, J-F ; L'éthique financière, Paris, PUF collection que sais-je ? numero 2604, 1991

Etchegoyen, A. ; Les entreprises ont-elles une âme? Pairs, Editions François Bourin, 1990

Even-Granboulan, G. ; Ethique et Economie. Quelle morale pour l'entreprise et le monde des affaires? Paris éditeur L'Harmattan, 1988 
Fayol, H. ; Administración industrial y general, Argentina, Librería "El Ateneo" Editorial, Traducción del francés de Dr. Dimitru, C. 1981 (1916)

Floris, B. ; La communication managériale. La modernisation symbolique des entreprises, Grenoble, PUG, 1996

Gélinier, O. ; L'éthique des affaires. Halte à la dérive, Paris, Editions du Seuil, 1991

Hardt, M. et Negri, A ; Empire, Paris Exils Editeur, traduit de l'américain par Canal, D-A, 2000

Koenig, G. ; “Développement de l'entreprise”, en Simon, Y. et Joffre, P. (sous la direction) ; Encyclopédie de Gestion, Paris, Economique, $2^{\mathrm{e}}$ édition, T. I, p. 938

; "Théorie économique de l'entreprise", en Simon, Y. et Joffre, P. (sous la direction) ; Encyclopédie de Gestion, Paris, Economique, $2^{\mathrm{e}}$ édition, T. III, p. 3310

Landier, H. ; Vers l'entreprise intelligente. Dynamique du changement et mutation du changement, Paris, Editions Calmann-Lévy, 1991

Le Tourneau, P. ; l'Ethique des affaires et du management au XXI siècle, Essai, Paris, éditeur Dalloz, 2000

Leroux, A. et Marciano, A. (sous la direction); Traité de philosophie économique, Paris, Economique, 1999

Leroy, E. ; "Quand le capital humain crée de la valeur", en La Tribune, Mardi 8 octobre 2002

Lipovetsky, G. ; Le crépuscule du devoir. L'éthique indolore des nouveaux temps démocratiques, Pais Éditions Gallimard, nrf essais, 1992

Louart, P. ; "Motivation", en Simon, Y. et Joffre, P. (sous la direction) ; Encyclopédie de Gestion, Paris, Economique, 2édition, T. II, p. 2095 
March, J.C. et Simon, H.A.; Organizations, New York, Wiley, (1957)

Marmuse, C. ; "Performance", en Simon, Y. et Joffre, P. (sous la direction) ; Encyclopédie de Gestion, Paris, Economique, 2 édition, T. II, p. 2194

Marx, K. ; Manuscrits parisiens, Paris, Bibliothèque de la Pléiade. Euvres III Philosophie, édition établie, présentée et annotée par Maximilien Rubel. NRF Gallimard, 1968a (1844)

; Le manifeste communiste, Paris, Bibliothèque de la Pléiade. Euvres I Economie, édition établie, présentée et annotée par Maximilien Rubel. NRF Gallimard, 1965a (1848)

; Introduction générale à la critique de l'économie politique, Paris, Bibliothèque de la Pléiade. Euvres I Economie, édition établie, présentée et annotée par Maximilien Rubel. NRF Gallimard, 1965b (1857)

; Le Capital, livres I, II et III, Paris, Bibliothèque de la Pléiade. Euvres I Economie, édition établie, présentée et annotée par Maximilien Rubel. NRF Gallimard, 1965c (1867), 1968b (1885) (1894)

et Engels, F. ; L'Idéologie allemande, Paris, Bibliothèque de la Pléiade. Euvres III Philosophie, édition établie, présentée et annotée par Maximilien Rubel. NRF Gallimard, 1982 (1846)

; Théories sur la Plus-value (Livre IV du Capital) Paris, EDITIONS SOCIALES, 1995 (1861-1863)

Mathiot, J.; Adam, S ; Philosophie et économie, de la sympathie à l'échange, PUF, 1990

Mercier, S. ; L'éthique dans les entreprises, Paris, La Découverte, Collection Repères, 1999

Naudet, J-Y ; (dirigée), Ethique des affaires : de l'éthique de l'entrepreneur au droit des affaires, Collection Ethique et Déontologie, Editeur Librairie de l'Université d'Aix-en-Provence, 1997 
Novak, M. ; Une éthique économique. Les valeurs de l'économie de marché, Paris, Les Editions du Cerf, 1987 (1982)

Petkantchin, V. ; Les sentiments moraux font la richesse des nations. Moralité des comportements et moralité des procédures dans l'œuvre d'Adam Smith, Editeur Librairie de l'Université d'Aix-en-Provence, 1996

Pribram K. ; Les Fondements de la Pensée économique ; ECONOMICA, 1986

Reitter, R. ; "Culture et identité", en Simon, Y. et Joffre, P. (sous la direction) ; Encyclopédie de Gestion, Paris, Economique, $2^{\mathrm{e}}$ édition, T. I, p. 878

Roux, D et Soulié, D. ; "Nouvelles technologies de l'information et gestion de l'entreprise", en Simon, Y. et Joffre, P. (sous la direction) ; Encyclopédie de Gestion, Paris, Economique, $2^{\mathrm{e}}$ édition, T. II, p. 2140

Smith, A. ; Théorie des sentiments moraux, Traduction par Biziou, M, Gautier, C, Pradeau, J. ; Paris, PUF, 1999 (1759) ; Recherche sur la nature et les causes de la richesse des nations, Traduction de Garnier, G ; Paris GF. Flammarion édition, 1991 (1776)

Simon, Y. et Joffre, P. (sous la direction) ; Encyclopédie de Gestion, Paris, Economique, $2^{\mathrm{e}}$ édition, 1997, T, I, II, III

Solomon, R.C. et Hanson, K.R. ; La morale en affaires clé de la réussite, Paris, Éd. Organisation, 1989

Simon, H. A. ; Rational Decision Making in Business Organization, American Economic, Review (Vol. 69, 1979)

Taylor, F.W. ; Management científico, Spain, Editions Orbis, 5êm edition, Traduction par Arrufat, A. 1986 (1911)

Sen, A. ; Ethique et économie, et autres essais, Paris, PUF, traduit de l'anglais par Marnat, S ; 1986 
Weber, M. ; L'éthique protestante et l'esprit du capitalisme, Paris, éditeur, Plon, 1967

Williamson, O.E. ; Transaction Cost Economics: The Governance of Contractual Relations, Jounal of Law and Ecomomicx (No. 2, 1979) ; Les institutions de l'économie, Paris, InterEditions, 1994 